INPLASY

PROTOCOL

To cite: Yang et al. Efficacy of Different exercise doses on global cognition in patients with mild cognitive impairment: a network meta-analysis. Inplasy protocol 202170080. doi:

10.37766/inplasy2021.7.0080

Received: 24 July 2021

Published: 24 July 2021

Corresponding author: Junchao Yang

474007589@qq.com

Author Affiliation:

Beijing Sport University.

Support: 2018YFC2000600.

Review Stage at time of this submission: The review has not yet started.

Conflicts of interest: None declared.

\section{Efficacy of Different exercise doses on global cognition in patients with mild cognitive impairment: a network meta-analysis}

\author{
Yang, J1; Wang, M2; Dong, Y3; Chen, Y4; Li, X5; Lu, M6; Yi, L7; \\ $\mathrm{Xu}, \mathrm{C}^{8}$.
}

Review question / Objective: To systematically review the efficacy of exercise therapy on global cognition in patients with cognitive impairment $(\mathrm{Cl})$ by network meta-analysis (NMA) and provide reference for the precision exercise scheme.

Condition being studied: Cognitive impairment $(\mathrm{Cl})$ includes two common types: dementia and mild cognitive impairment (MCl). It is estimated that there are about 50 million people with dementia worldwide at present, and the total will reach 74.7 million by 2030. Dementia is one of the main causes of disability and dependency in the elderly. The cost of caring for dementia patients is expected to reach us $\$ 2$ trillion annually from 2030 . This imposes a heavy burden on society, especially in low - and middle-income countries where $60 \%$ of dementia patients live. $\mathrm{MCl}$ is a transitional state between normal cognitive function and dementia (or Alzheimer's disease). $14.9 \%$ of patients ( $\geq 65$ years old) with $\mathrm{MCl}$ develop dementia within 2 years, and $16 \%$ of patients with amnestic $\mathrm{MCl}(\mathrm{aMCl})$ develop Alzheimer's disease. However, only a small number of $\mathrm{MCl}$ patients $(14 \sim 40 \%)$ remain stable or return to normal. Unfortunately, there are no drugs that can cure dementia and $\mathrm{MCl}$.

INPLASY registration number: This protocol was registered with the International Platform of Registered Systematic Review and Meta-Analysis Protocols (INPLASY) on 24 July 2021 and was last updated on 24 July 2021 (registration number INPLASY202170080).

\section{INTRODUCTION}

Review question / Objective: To systematically review the efficacy of exercise therapy on global cognition in patients with cognitive impairment $(\mathrm{Cl})$ by network meta-analysis (NMA) and provide reference for the precision exercise scheme. 
Condition being studied: Cognitive impairment $(\mathrm{Cl})$ includes two common types: dementia and mild cognitive impairment $(\mathrm{MCl})$. It is estimated that there are about $\mathbf{5 0}$ million people with dementia worldwide at present, and the total will reach 74.7 million by 2030 . Dementia is one of the main causes of disability and dependency in the elderly. The cost of caring for dementia patients is expected to reach us \$2 trillion annually from 2030 . This imposes a heavy burden on society, especially in low - and middle-income countries where $60 \%$ of dementia patients live. $\mathrm{MCl}$ is a transitional state between normal cognitive function and dementia (or Alzheimer's disease). $14.9 \%$ of patients ( $\geq 65$ years old) with $\mathrm{MCl}$ develop dementia within 2 years, and $16 \%$ of patients with amnestic $\mathrm{MCl}$ (aMCl) develop Alzheimer's disease. However, only a small number of $\mathrm{MCl}$ patients $(14 \sim 40 \%)$ remain stable or return to normal. Unfortunately, there are no drugs that can cure dementia and $\mathrm{MCl}$.

\section{METHODS}

Search strategy: Two review authors (JC and MY) independently searched PubMed, EMbase, The Cochrane Library, China National Knowledge Infrastructure (CNKI), Wanfang Digital Periodicals (WANFANG), from inception to January May 2021 to identify potentially eligible studies.Three groups of search terms including Cognitive, Cognition, Cognitive Dysfunction, Mild Cognitive Mpairment, Dementia, Alzheimer, Mild Cognitive Disorder, Aerobic, Walking, High Intensity Interval, Resistance, Core Stability, Dance, Breathing Exercise, Virtual Reality Exercise, Whole Body Vibration Exercise, Stretching, Body-Mind Exercise, Yoga, Pilates, Tai Chi, Taijiquan, Health Qigong, Yijinjing , Wuqinxi, Liuzijue, Baduanjin, Multicomponent Exercise, Randomized, Randomized Controlled Trial were combined for search. In addition, the references are retroactively included in the literature in order to supplement and obtain the relevant literature.

Participant or population: Cognitive impairment.
Intervention: The intervention was planned, organized, and repeated exercise therapy, either alone or on a control basisexercise.

Comparator: The control group did not have any intervention, maintain the original lifestyle, health education or stretching.For dementia patients, the intervention group also included RCTs with exercise in addition to conventional treatment.

Study designs to be included: Randomized controlled trials.

Eligibility criteria: (1) Randomized controlled trial (RCTs). (2) The elderly ( $\geq 50$ years old), including patients with mild cognitive impairment and dementia, refer to Petersen's diagnostic criteria, DIAGNOSTIC and Statistical Manual of Mental Disorders. The diagnostic and statistical manual of mental disorders and the diagnostic process of cognitive impairment in elderly Chinese Psychiatric Association were recommended to be included in the study. (3) The intervention was planned, organized, and repeated exercise therapy, either alone or on a control basis. The control group did not have any intervention, maintain the original lifestyle, health education or stretching.For dementia patients, the intervention group also included RCTs with exercise in addition to conventional treatment. Comparisons of the above interventions will also be included. (4) trials with one or more of the following outcome indicators can be included. The main outcome indicators include: overall cognitive function.

Information sources: The PubMed, EMbase, The Cochrane Library, Web of Science, CNKI and WanFang Data databases.

Main outcome(s): Global cognition.

Quality assessment / Risk of bias analysis: The bias risk of the included studies was evaluated by 2 evaluators in accordance with the Cochrane Manual for RCT bias risk assessment tool, and 7 aspects were evaluated. These include randomization of 
assignment methods, hiding of assignment protocols, blinding of study protocol and treatment protocol implementors, blinding of study outcome measures, integrity of outcome data, selective reporting of results, and other sources of bias.

Strategy of data synthesis: In this paper, ReMan 5.3 and Stata 15.1 software were used for meta-analysis. In this study, outcome indicators were continuous variables, MD or SMD and $95 \% \mathrm{Cl}$ were used as effect size indicators, and $I^{\wedge} 2$ was used to determine the heterogeneity. If $I^{\wedge} 2 \geq 50 \%$, subgroup analysis was performed.If data conversion and merging are required for some literatures, the calculator in ReMan $\mathbf{5 . 3}$ is used. Use node analysis to perform inconsistency check. If $>0.05$, the consistency model was adopted for analysis.At the same time, node splitting method is used to test local inconsistency. When direct and circumstantial evidence are inconsistent, direct comparisons are made using ReMan 5.3. The ranking index is reflected by surface under the cumulative ranking (SUCRA), in which $0 \leq$ SUCRA $\leq 100 \%, 100 \%$ represents the most effective exercise therapy, and 0 is the worst and invalid.

Subgroup analysis: If $I^{\wedge} 2 \geq 50 \%$, subgroup analysis was performed.

Sensitivity analysis: This analysis is performed if there are low-quality studies.

Country(ies) involved: China.

Keywords: Exercise; mild cognitive impairment; dementia; global cognition; Network meta-analysis.

Contributions of each author:

Author 1 - Junchao Yang.

Author 2 - Mengyue Wang.

Author 3 - Yunfeng Dong.

Author 4 - Yuyang Chen.

Author 5 - Xinyuan Li.

Author 6 - Mingyue Lu.

Author 7 - Longyan Yi.

Author 8 - Chunyan Xu. 